\title{
Have Necessary Healthcare Personnel or Equipment
}

National Cancer Institute

\section{Source}

National Cancer Institute. Have Necessary Healthcare Personnel or Equipment. NCI

Thesaurus. Code C128497.

The healthcare provider or setting must have particular medical equipment or personnel with particular training on-site. 